\title{
El control interno y la gestión del Sistema Integral de Salud en el Hospital Regional Docente Materno Infantil "El Carmen"
}

\section{The internal control and management of the Comprehensive Health System in the Hospital Regional Docente Materno Infantil "El Carmen"}

\author{
'Munguía Palacios, Rosa Violeta; García Puente, David Javier \\ Facultad de Contabilidad, Universidad Nacional del Centro del Perú \\ Email: rmunguia@uncp.edu.pe
}

\section{Resumen}

La presente investigación, titulada "El control interno y la gestión del Sistema Integral de Salud en el Hospital Regional Docente Materno Infantil", estudia un establecimiento de Salud III-E, el cual se alinea a uno de los objetivos del milenio. El servicio surge como prioridad, ante una nueva forma de atención bajo la modalidad del Sistema Integral de Salud, ampliando el universo de subsidios a los beneficiarios; atenciones que se convierten en una nueva fuente de financiamiento. Es importante ahora, dado que significa un $98 \%$ de pacientes cubiertos.

Se hace necesario el manejo, supervisión y manejo del control interno, a fin de incidir en la consecución de objetivos hospitalarios basados en mejorar la cobertura con una eficiente atención al usuario, incrementar los desembolsos por transferencias del SIS, que se convierten en asignaciones presupuestales, basados en el histórico que a partir del 2012 ha crecido en razón al 115 \% hasta el año 2015.

Es una investigación aplicada, donde se trabajó en las actividades propias del área, identificando cada componente del control en la gestión del Sistema Integral de Salud del hospital; a través del diagnóstico de los elementos del sistema, en las tareas del Área de Seguros y Servicios conexos, mediante aplicación de normas, revisión de documentos de gestión, aplicación de cuestionarios y guías de entrevistas a los funcionarios y servidores, a fin de obtener la respuesta de los trabajadores, la misma que sirvió para el análisis de datos de las variables cualitativas a través de la observación, el análisis de contenido, llegando a la conclusión que el nivel de implementación de las normas del control están asociados a los niveles de cumplimiento de los componentes del mismo sistema de control interno en los procedimientos del SIS. En consecuencia, el cumplimiento total de las normas es importante y por ende sus actividades mejoran, obteniéndose una perspectiva clara de la situación actual; pudiéndose detectar las brechas de gestión y proponer mejoras sustanciales en el manejo hospitalario de las diferentes áreas involucradas.

Palabras Claves: control interno, gestión del Sistema Integral de Salud, componentes de control, normas de control, transferencias, subsidios, asignación presupuestal.

\section{Abstract}

The present investigation, named "Internal control and management of the Sistema Integral de Salud in the Hospital Regional Docente Materno Infantil”, studies a health facility III-E, which is aligned with one of the objectives of the millennium. The service emerges as a priority, in fornt of a new care form under the modality of the Sistema de Salud Integral, widening the subsidies universe to beneficiaries; attentions that become a new funding source. It is important now, since it means $98 \%$ of covered patients.

It is necessary to supervise and manage the internal control, to incide in the achievement of hospital objectives based on improving coverage with an efficient user service, increase disbursements due to SIS transfers, which are converted into budget allocations, historically based on the fact that since 2012 it has grown on a reason of $115 \%$ until 2015.

It is an applied research, where the area activities were worked on, identifying each control component in the management of the Sistema Integral de Salud of the hospital; through diagnosing of system elements, in the tasks of the Insurance and Related Services Area, through the application of norms, review of management documents, application of questionnaires and interview guides to officials and servers, in order to obtain the workers response, the same which served for the analysis of data of the qualitative variables through observation, content analysis, reaching the conclusion that the implementation level of the control norms are associated with compliance levels of the components of the same internal control system in the SIS procedures. Consequently, full compliance with the nomrs is important and therefore their activities improve, obtaining a clear perspective of the current situation; It is possible to detect management gaps and propose substantial improvements in the hospital management of the different areas involved

Keywords: internal control, Integral Health System management, control components, control rules, transfers, subsidies, budget allocation. 


\section{Introducción}

Las entidades hospitalarias públicas del Ministerio de Salud en Huancayo, presentan múltiples problemas respecto al desempeño laboral de sus colaboradores en el cumplimiento de actividades y labores encomendadas para el logro de objetivos institucionales. Teniendo como marco normativo el Plan Estratégico Institucional de la Región Junín 2015-2017 y, siendo uno de los componentes el derecho humano y dignidad, el "Desarrollo Humano", “pobreza” y "ciudadanía democrática”, donde los más pobres accedan a los servicios básicos y, así mejorar el acceso a los mismos para el desarrollo de capacidades respecto a la calidad de prestación de salud para la población; por ello, se crea el Sistema Integral de Salud el mismo que llega a la población más vulnerable inicialmente y se va masificando, convirtiendo a las atenciones realizadas en una fuente de financiamiento directa a través del Seguro Integral de Salud. Es necesario plantear un estudio para que mejoren los factores determinantes para una eficiente gestión que incremente y recupere al $100 \%$ las atenciones brindadas en cada uno de los servicios del Hospital Regional Docente Materno Infantil "El Carmen”, donde a la fecha no se implantan los procedimientos propios de control interno que permitan llegar a mejorar la gestión de la Oficina de Seguros, responsable del manejo del SIS y no se ha trabajado técnicamente en lo referente a controles administrativos, a pesar de ser una necesidad la implementación de los componentes prioritarios de la Ley de Control Interno, para la correcta gestión en los recursos y servicios que se brinda a través del SIS.

La implementación del control interno a través de las guías emitidas por el ente rector CGR, como proceso integral efectuado por la dirección y el personal comprometido en la consecución de la misión del hospital, la ejecución ordenada, ética, económica, eficiente y efectiva de las operaciones, el cumplimiento de las obligaciones de responsabilidad y la normatividad aplicable; es una necesidad que nos permite salvaguardar los recursos evitando pérdidas, mal uso y daño en el manejo del SIS del hospital, permitiéndo un mejor manejo de los recursos en lo que se refiere a captación y gastos. En el estudio se evidencia que hay deficiencias durante el proceso de reconocimiento de deudas generadas por atenciones a través del SIS. Al respecto, se ha determinado que las historias clínicas presentan errores en su codificación y conceptos, lo que limita el descargo de datos, información incorrecta, se consideran rubros no reconocidos por el SIS; así como, la deficiencia en las adquisiciones de bienes y servicios, dado que el dinero llega de un momento a otro de manera acumulada y los procesos no son suficientes ni competentes. Acciones que son desfavorables para alcanzar las metas y objetivos institucionales, afectando a los pacientes, quienes provienen de familias de bajos recursos. El control interno gubernamental en los sistemas administrativos del sector público, esta normado en todos sus niveles; encontrándose en la actualidad acondicionadas a las normas dictadas en materia de control, se vuelve relevante porque las instituciones hospitalarias, para este caso, necesitan urgentemente aumentar su credibilidad en la sociedad para asegurar el desarrollo de los más necesitados, atrayendo una mayor inversión y por ende el crecimiento del país.

Para mejorar la calidad de los servicios de salud de la región Junín, que es uno de los objetivos estratégicos y por ende se convierte en reto el mejorar la gestión de toda organización, es de necesidad el compromiso de funcionarios y trabajadores del área de seguros el implementar el control interno en la gestión del Sistema Integral de Seguros del HRDMIEC, para la aplicación de las diferente normas, directivas y protocolos a ser implementadas, con sentido de eficiencia, eficacia y economía de los recursos asignados; así como, estandarizando los procesos tendientes a elevar los niveles de productividad, propiciando un clima laboral motivante y retador que lleven a los empleados desarrollar el trabajo en equipo y, por ende, elevar los montos a través de transferencias presupuestales por las atenciones dadas a través el Sistema Integral de Salud como mayor fuente de recaudación.

\section{Materiales y métodos}

En esta investigación se ha utilizado el método descriptivo para describir, analizar e interpretar la evolución de la atención del Sistema Integral de Salud en el hospital; el mismo que nos llevó a encontrar los cismas que existen en la actual gestión que tienen que ver directamente con la implementación de los lineamientos de control interno, el cual ayudará a una eficiente gestión de los recursos. Asimismo, el método analítico - sintético, que permite analizar la situación actual del Sistema Integral de Salud en el Hospital Regional Docente Materno Infantil "El Carmen", llegando a identificar el problema del estudio al descomponer en partes e identificar con mayor precisión, relacionando y creando explicaciones de la importancia de la aplicación de la normatividad del control Interno. El método inductivo - Deductivo llevó a inducir que aplicando los cuestionarios con algunos ítems de los componentes del control interno a partir del diagnóstico, surgen brechas que deberán ser subsanadas a través de la implementación de las normas que respondan a cada proceso que se convierte en una necesidad para que los trabajadores del Hospital Regional Docente Materno Infantil "El Carmen, cometan menos errores y brinden una mejor atención al usuario y consecuentemente se optimice los desembolsos del SIS. Con la información obtenida se dedujo que el resto de hospitales tienen los mismos problemas, dado que el SIS genera la mayor cantidad de pacientes atendidos y por ende mayores recursos. A través 
del método de observación se mantuvo el contacto con los trabajadores asistenciales, personal administrativo, profesionales de la salud y directivos del Hospital Regio- nal Docente Materno Infantil "El Carmen” Por lo que se percibe que existen muchos problemas por falta de la implementación de control interno en toda la organización.

Cuadro $\mathrm{N}^{\circ} 1$

\begin{tabular}{|c|c|c|}
\hline Las Variables & Los Indicadores & Medidas \\
\hline \multirow{4}{*}{$\begin{array}{l}\text { Variable independiente: } \\
\qquad \mathrm{El} \mathrm{Cl}\end{array}$} & \multirow{4}{*}{ Normas de $\mathrm{Cl}$ gubernamentales } & Elementos del Control Interno. \\
\hline & & Niveles de Control. \\
\hline & & Guias \\
\hline & & Resoluciones \\
\hline \multirow{12}{*}{$\begin{array}{l}\text { Variable dependiente: } \\
\text { Gestión del Sistema Integral de Salud }\end{array}$} & \multirow{2}{*}{ Selección de Beneficiarios. } & Registros, empadronamiento \\
\hline & & Referencia \\
\hline & \multirow{5}{*}{ Atención Usuarios. } & Cupo de triaje. \\
\hline & & Formato Unico de atención FUT. \\
\hline & & Admisión Historia Clìnica \\
\hline & & Selección Consultorios. \\
\hline & & Examenes ayuda diagnóstico \\
\hline & \multirow{3}{*}{ Recuperación fondos } & Auditorias \\
\hline & & ARFSIS \\
\hline & & UDRJ \\
\hline & \multirow{2}{*}{ Monitoreo, Seguimiento y Supervisión } & Supervisión interna. \\
\hline & & Supervisión externa SUSALUD \\
\hline
\end{tabular}

\section{Cuadro $\mathrm{N}^{\circ} 2$}

Población HRDMI El Carmen

\begin{tabular}{lc}
\hline \multicolumn{1}{c}{ PERSONAL DEL HOSPITAL SEGÚN CAP } & TOTAL \\
\hline \hline Funcionarios y directivos & 06 \\
\hline \hline Médicos & 43 \\
\hline \hline Profesionales de la salud & 159 \\
\hline \hline Personal técnico asistencial & 153 \\
\hline \hline Personal auxiliar asistencial & 2 \\
\hline \hline Personal profesional administrativo & 17 \\
\hline \hline Personal técnico administrativo & 31 \\
\hline \hline Total & 411 \\
\hline \hline
\end{tabular}

Muestra: Aplicamos la siguiente formula:

$$
n=\frac{p \cdot q Z^{2} \cdot N}{E E^{2} N-1 \pi p \cdot q Z^{2}}
$$

$\mathrm{n}=(0.5 \times 0.5 \times(1.96) 2 \times 411) /(((0.09) 2 \times 410)+$ $(0.5 \times 0.5 \times(1.96) 2))$

$\mathrm{n}=90$

Guia de Entrevista: Se ha entrevistado al personal del Hospital Regional Docente Materno Infantil "El Carmen", a los que manejan información desde la captación del paciente y los sistemas administrativos y operativos involucrados en su atención

Encuesta: Para tener datos reales sobre el tema de investigación, se ha elaborado una cantidad de cuestionarios, de los cuales, solo se llegó a utilizar un cuestionario, por cuestiones de tiempo, y es el que se ha aplicado. Los cuestionarios no utilizados fueron excluídos, porque al aplicarse al personal, estos no concluyeron por ser muy extenso. El cuestionario final de la encuesta, se ha aplicado al total de personal que labora en la Oficina de Seguros que suman 28; así como, los que cumple funciones de jefaturas en las diferentes Oficinas y Departamentos finales del Hospital Regional Docente Materno Infantil "El Carmen”. Se ha elaborado los cuestionarios de control interno para toda la gestión porque el sistema integral de salud si bien es una fuente de financiamiento depende de cada trabajador y cada área para que los recursos provenientes de la atención por SIS sea manejada en cumplimiento a las normas y beneficiando a los usuarios

Observación: Permanentemente en contacto con el personal mencionado líneas arriba, quienes compartieron las debilidades más frecuentes con que cuentan dichas oficinas, todas relacionadas con la implementación, control interno y las atenciones del SIS 


\section{Resultados}

Presentación, Análisis e Interpretación de los Datos: Para nuestra investigación las preguntas se realizaron en función a cuatro alternativas ante los cuales se solicita la respuesta (SI, NO, TAL VEZ y NO SABE/NO OPINA) de la persona encuestada. Para el estudio se elaboró un cuestionario de diecisiete preguntas, que fue aplicado a la muestra correspondiente. Es decir, se encuestaron a 90 trabajadores del Hospital Regional Docente Materno Infantil, siendo 28 el total de trabajadores del SIS y la diferencia entre Directores, ejecutivos, personal profesional de la salud, técnico y administrativo.

Variable Independiente: Control Interno.

\section{Pregunta 1}

¿Conoce la importancia del sistema de control?

\begin{tabular}{lcrr} 
& Frecuencia & Porcentaje & $\begin{array}{r}\text { Porcentaje } \\
\text { acumulado }\end{array}$ \\
\hline \hline $\mathrm{SI}$ & 42 & 46,7 & 46,7 \\
\hline NO & 24 & 26,7 & 73,3 \\
\hline \hline TAL VEZ & 6 & 6,7 & 80,0 \\
\hline \hline $\begin{array}{l}\text { NO SABE/ } \\
\text { NO OPINA }\end{array}$ & 18 & 20,0 & 100,0 \\
\hline \hline Total & $\mathbf{9 0}$ & $\mathbf{1 0 0 , 0}$ & \\
\hline \hline
\end{tabular}

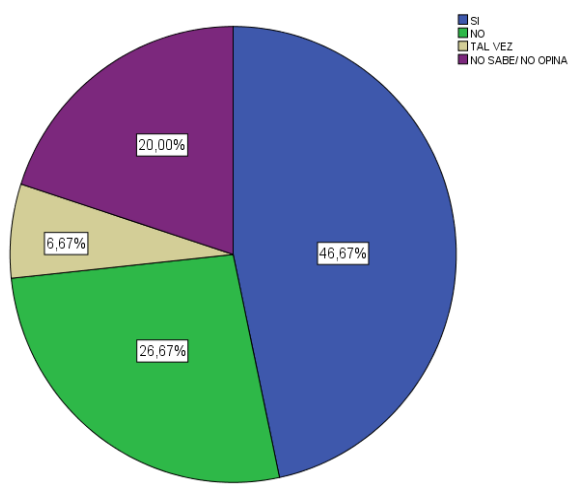

El $46.67 \%$ de los encuestados, opinan que conocen de la importancia del sistema de control dentro de las instituciones, un $26.67 \%$ restante no conoce, el $20 \%$ no sabe/ no opina y el $6.67 \%$ tal vez.

Pregunta 2

¿Sabe si se aplican los elementos del CI?

\begin{tabular}{lccc} 
& Frecuencia & Porcentaje & $\begin{array}{c}\text { Porcentaje } \\
\text { acumulado }\end{array}$ \\
\hline \hline $\mathrm{SI}$ & 42 & 46,7 & 46,7 \\
\hline \hline NO & 18 & 20,0 & 66,7 \\
\hline \hline TAL VEZ & 12 & 13,3 & 80,0 \\
\hline \hline $\begin{array}{l}\text { NO SABE/ } \\
\text { NO OPINA }\end{array}$ & 18 & 20,0 & 100,0 \\
\hline \hline Total & $\mathbf{9 0}$ & $\mathbf{1 0 0 , 0}$ & \\
\hline \hline
\end{tabular}

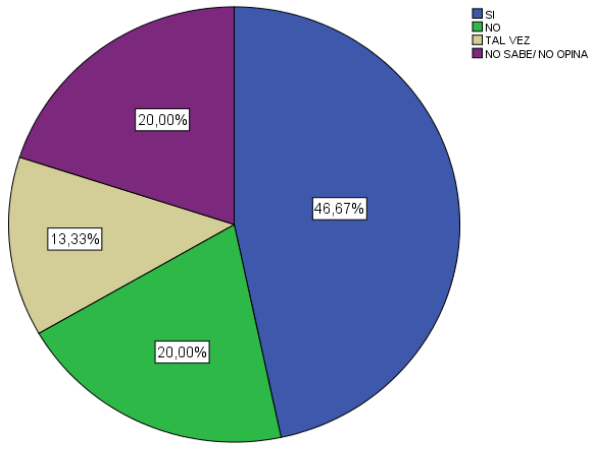

Se aprecia que el $46.67 \%$ de los encuestados opinan que si conocen que se aplican los elementos de control interno, un $20 \%$ no sabe, el $20 \%$ no sabe/ no opina y el $13 \%$ tal vez.

\section{Pregunta 3}

¿Conoce si se viene aplicando el control interno previo y simultáneo en el hospital?

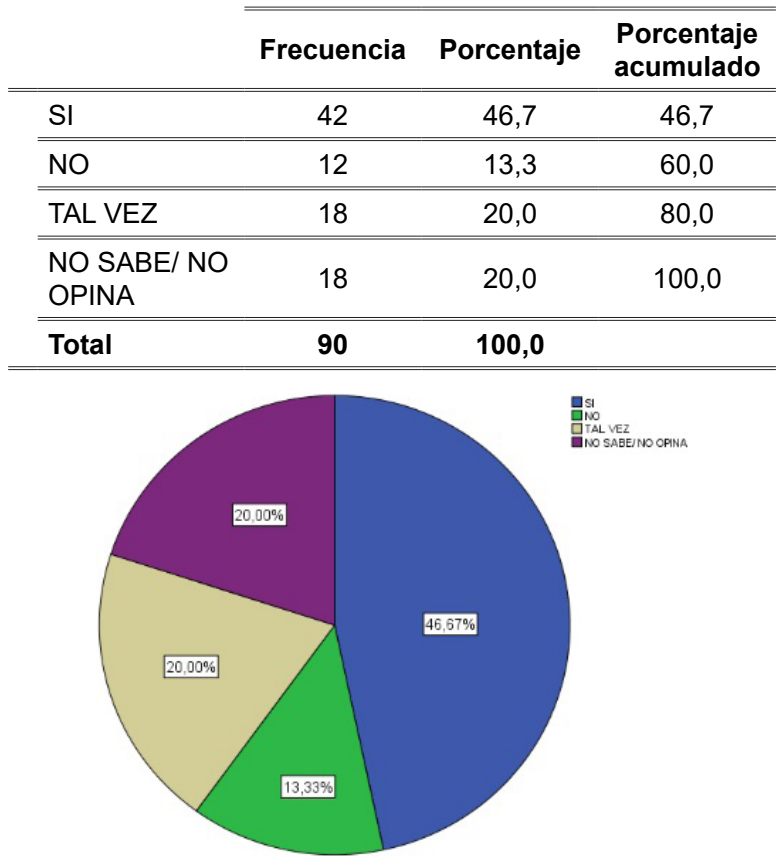

se observa que el $47 \%$ de los encuestados opinan que si tienen conocimiento que se viene aplicando el control interno previo y simultáneo, un $13 \%$ restante no lo tiene, el $20 \%$ no sabe/ no opina y el $20 \%$ tal vez.

\section{Pregunta 4}

¿Sabe que el CI ayuda al cumplimiento de las leyes y regulaciones aplicables?

\begin{tabular}{lccc} 
& Frecuencia & Porcentaje & $\begin{array}{c}\text { Porcentaje } \\
\text { acumulado }\end{array}$ \\
\hline \hline $\mathrm{SI}$ & 48 & 53,3 & 53,3 \\
\hline $\mathrm{NO}$ & 18 & 20,0 & 73,3 \\
\hline \hline TAL VEZ & 12 & 13,3 & 86,7 \\
\hline \hline $\begin{array}{l}\text { NO SABE/ NO } \\
\text { OPINA }\end{array}$ & 12 & 13,3 & 100,0 \\
\hline \hline Total & $\mathbf{9 0}$ & $\mathbf{1 0 0 , 0}$ & \\
\hline \hline
\end{tabular}




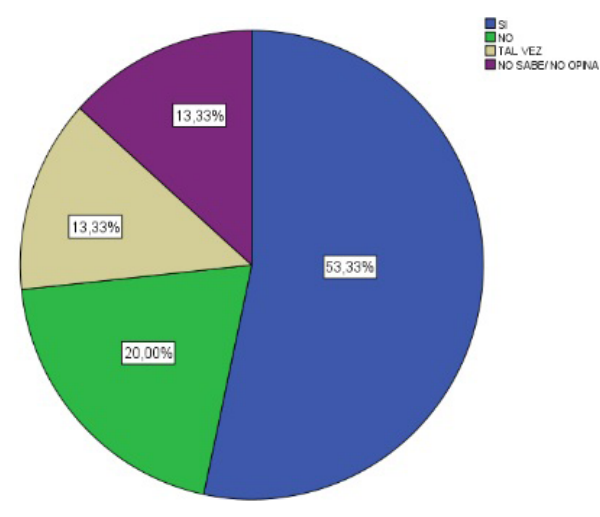

En este gráfico, vemos que el $53.33 \%$ de los encuestados manifiestan que si tienen conocimiento que el CI ayuda al cumplimiento de las leyes y regulaciones aplicables, un $20 \%$ restante no lo tiene, el $13 \%$ no sabe/ no opina y el 13\% tal vez.

\section{Pregunta 5}

¿Cree que el CI ayuda a la ejecución ordenada, ética, económica, eficiente y efectiva de las operaciones?

\begin{tabular}{lccc} 
& Frecuencia & Porcentaje & $\begin{array}{c}\text { Porcentaje } \\
\text { acumulado }\end{array}$ \\
\hline \hline $\mathrm{SI}$ & 54 & 60,0 & 60,0 \\
\hline \hline NO & 18 & 20,0 & 80,0 \\
\hline \hline TAL VEZ & 6 & 6,7 & 86,7 \\
\hline \hline $\begin{array}{l}\text { NO SABE/ NO } \\
\text { OPINA }\end{array}$ & 12 & 13,3 & 100,0 \\
\hline \hline Total & $\mathbf{9 0}$ & $\mathbf{1 0 0 , 0}$ & \\
\hline \hline
\end{tabular}

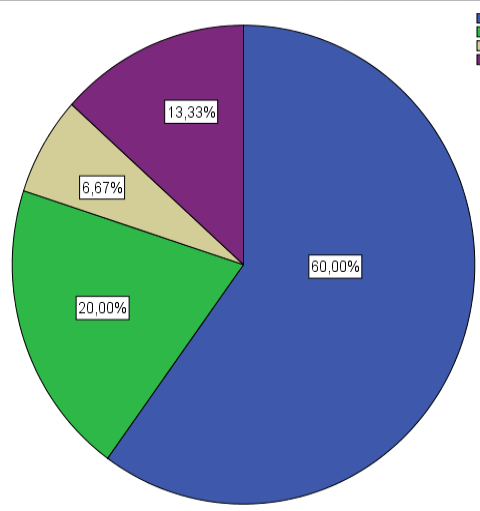

El $60 \%$ opina que si tienen conocimiento que el CI ayuda a la ejecución ordenada, ética, económica, eficiente y efectiva de las operaciones, un $20 \%$ restante desconoce, el $13 \%$ no sabe/ no opina y el $7 \%$ tal vez.

\section{Pregunta 6}

¿Cree que la aplicación del CI ayuda a salvaguardar los recursos de evitar pérdidas, mal uso y daño?

\begin{tabular}{lccc}
\cline { 2 - 4 } & Frecuencia & Porcentaje & $\begin{array}{c}\text { Porcentaje } \\
\text { acumulado }\end{array}$ \\
\hline \hline SI & 48 & 53,3 & 53,3 \\
\hline \hline NO & 18 & 20,0 & 73,3 \\
\hline \hline TAL VEZ & 12 & 13,3 & 86,7 \\
\hline \hline NO SABE/ NO & 12 & 13,3 & 100,0 \\
OPINA & $\mathbf{9 0}$ & $\mathbf{1 0 0 , 0}$ & \\
\hline \hline Total & &
\end{tabular}

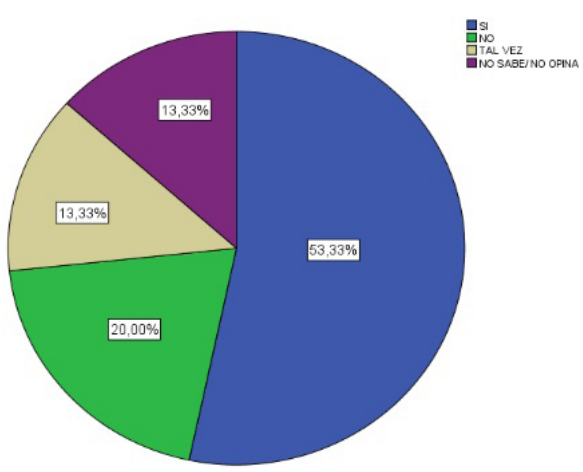

Se observa que el $53 \%$ de los encuestados afirman que el CI ayuda a salvaguardar los recursos a fin de evitar pérdidas, mal uso y daño, un $20 \%$ restante no, el $13 \%$ no sabe/ no opina y el $13 \%$ tal vez.

\section{Pregunta 7}

¿Cree que las normas de CI conducen al eficiente funcionamiento de toda gestión?

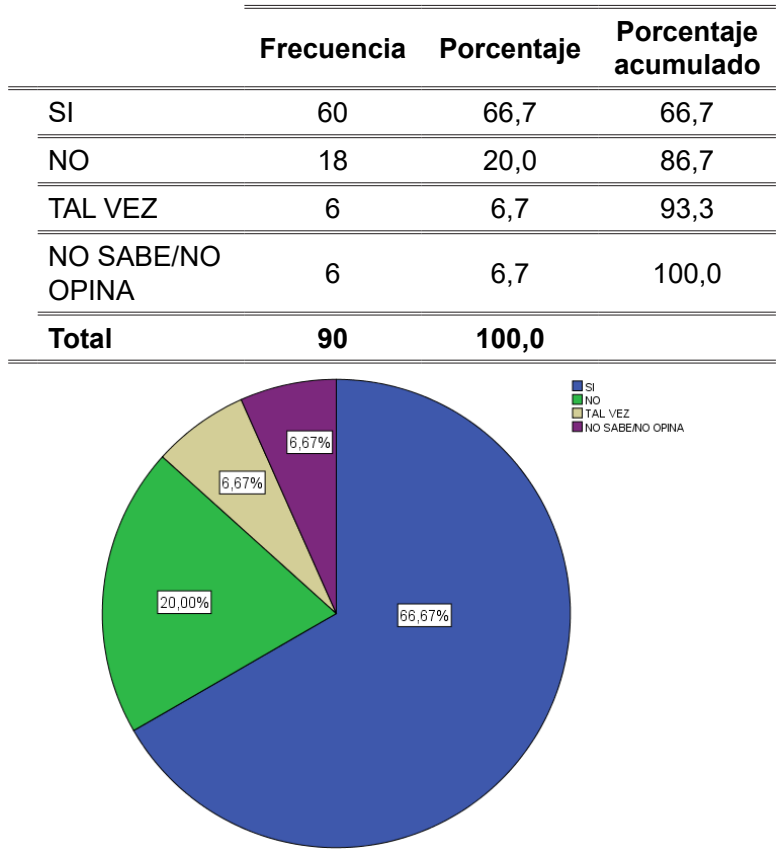

Aquí se demuestra que el $67 \%$ de los encuestados afirman que las normas de control interno conducen al eficiente funcionamiento de toda gestión, un $20 \%$ restante no, el $7 \%$ no sabe/ no opina y el $7 \%$ tal vez.

Variable Dependiente: Gestión del Sistema Integral de Salud SIS.

\section{Pregunta 8}

¿Cree usted que la captación de los beneficiarios en el primer nivel de atención del SIS permite seleccionar a beneficiarios vulnerables en condición de pobreza y pobreza extrema? 


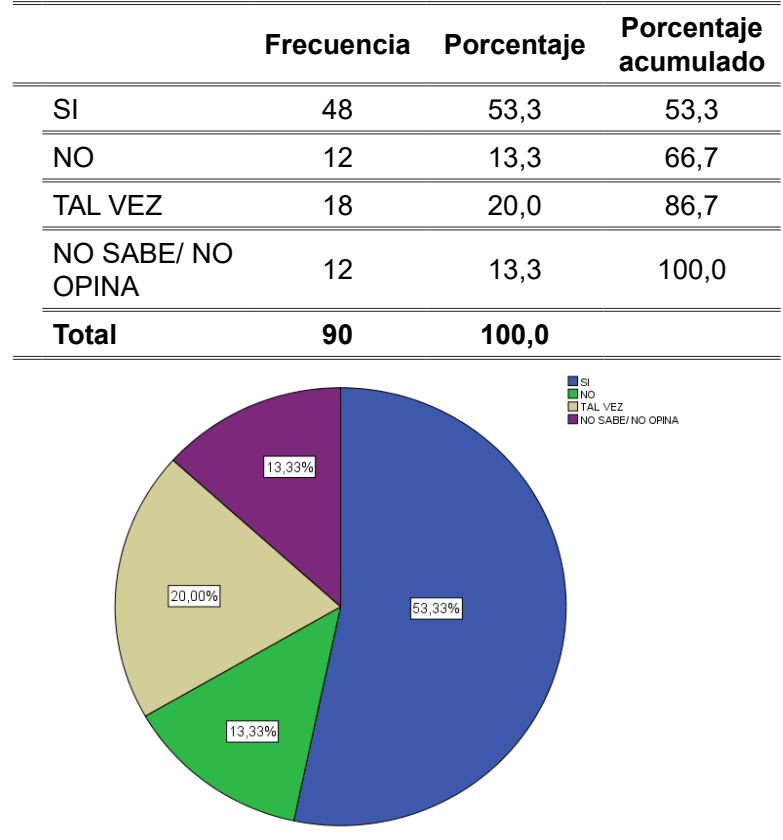

La encuesta demuestra que el $54 \%$ de los encuestados afirman que la captación realizada a beneficiarios en los primeros niveles de atención del SIS (Puestos y Centros de Salud) permite enfocar la atención a los pobladores más vulnerables, un $13 \%$ restante dice que no, el $13 \%$ no sabe/ no opina y el $20 \%$ tal vez.

\section{Pregunta 9}

Observa que el registro de los padrones del PGH (Padrón General de Hogares) y el SISFOH

(Sistema de Focalización de Hogares) están al día?

\begin{tabular}{lccc}
\cline { 2 - 4 } & Frecuencia & Porcentaje & $\begin{array}{c}\text { Porcentaje } \\
\text { acumulado }\end{array}$ \\
\hline $\mathrm{SI}$ & 54 & 60,0 & 60,0 \\
\hline NO & 12 & 13,3 & 73,3 \\
\hline \hline TAL VEZ & 18 & 20,0 & 93,3 \\
\hline \hline $\begin{array}{l}\text { NO SABE/ NO } \\
\text { OPINA }\end{array}$ & 6 & 6,7 & 100,0 \\
\hline \hline Total & $\mathbf{9 0}$ & $\mathbf{1 0 0 , 0}$ & \\
\hline \hline
\end{tabular}

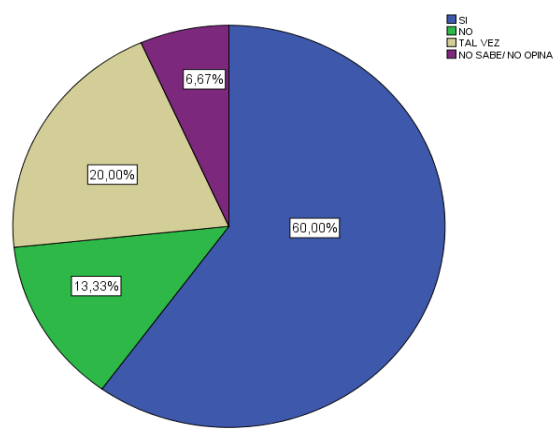

Aquí vemos que el $60 \%$ de los trabajadores afirman que los datos en los padrones del PGH y del SISFOH son actuales, un $13 \%$ restante no, el $7 \%$ no sabe/ no opina y el $20 \%$ tal vez.

\section{Pregunta 10}

¿Cree que la actualización y emisión de normas internas mejoraron la gestión del SIS?

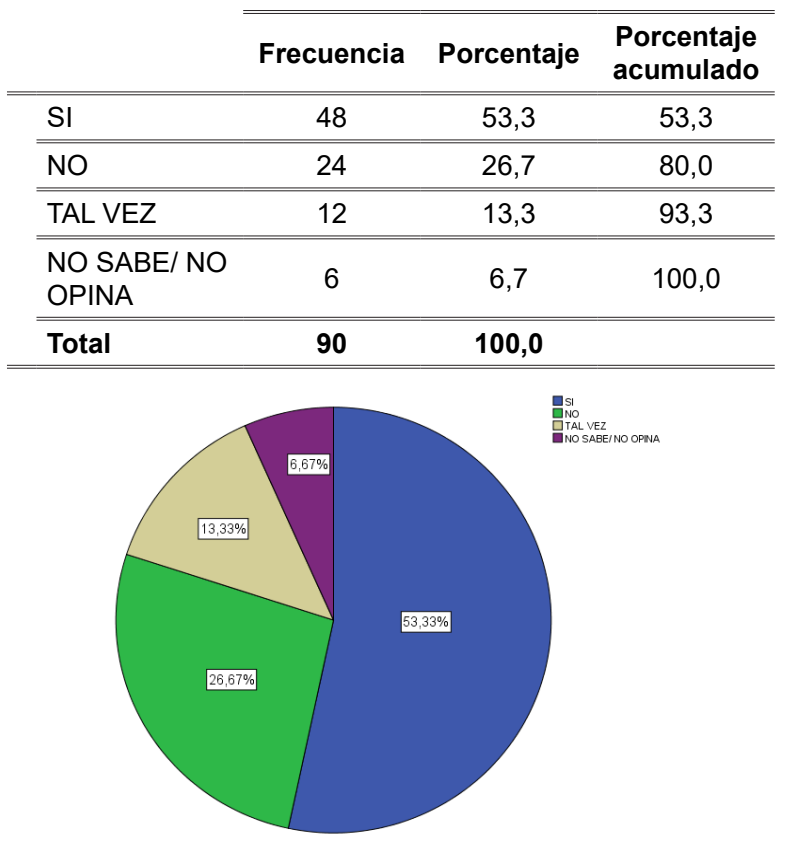

El $53 \%$ de los encuestados afirman que la actualización y emisión de normas internas mejoraron la gestión del SIS, un $27 \%$ restante no, el $7 \%$ no sabe/ no opina y el $13 \%$ tal vez.

\section{Pregunta 11}

¿Cree que el fortalecimiento de capacidades técnicas de los trabajadores del SIS contribuyó a fortalecer el control previo y el simultáneo?

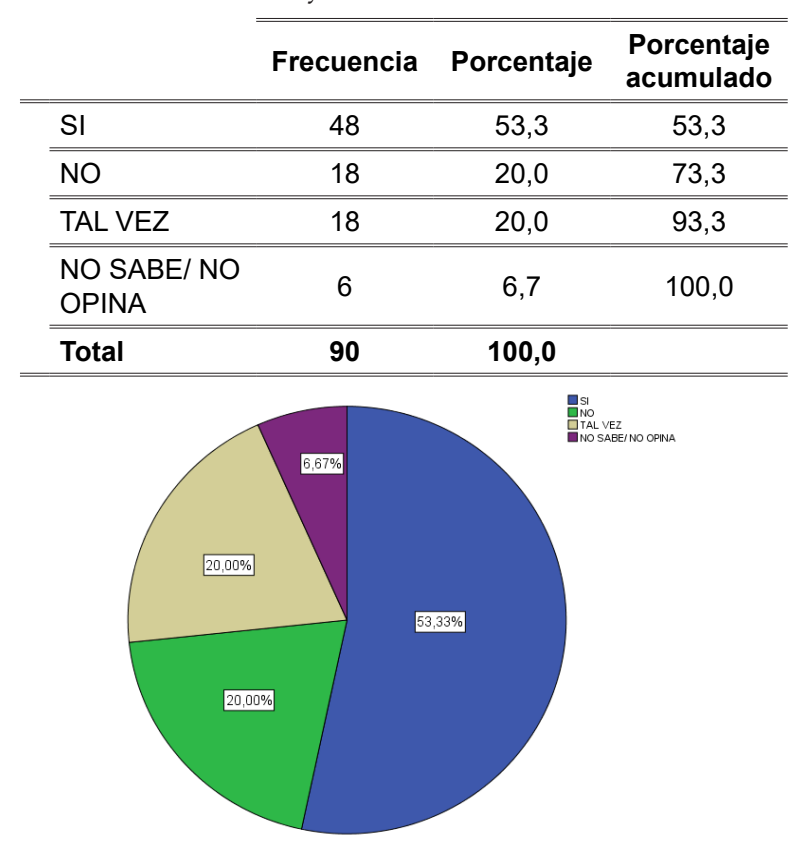

Se observa que el $53 \%$ de los encuestados afirman que el fortalecimiento de capacidades técnicas de los trabajadores del SIS contribuyó a fortalecer el control previo y simultáneo, un $20 \%$ restante no, el $7 \%$ no sabe/ no opina y el $20 \%$ tal vez. 


\section{Pregunta 12}

¿Cree que la aplicación de auditorías a las Historias Clínicas diseñado para el SIS permiten incrementar los reembolsos?

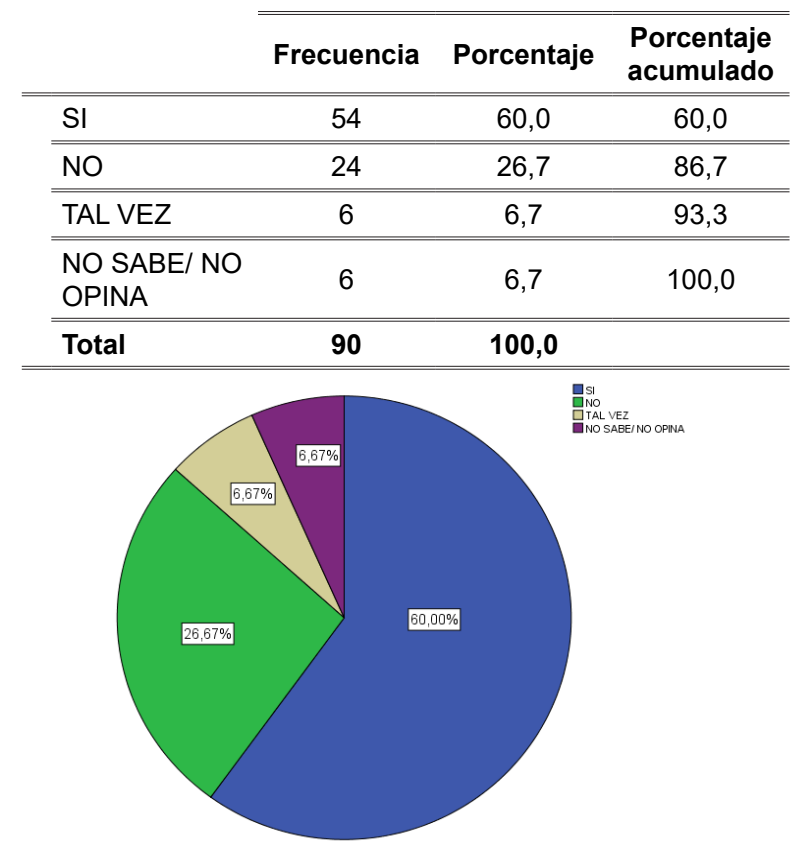

Se observa que el $60 \%$ de los encuestados afirman que la aplicación de controles internos diseñado en almacén ayudaron a mejorar el control del mismo, un $27 \%$ restante no, el $7 \%$ no sabe/ no opina y el $7 \%$ tal vez.

\section{Pregunta 13}

¿Observa que el tipo de atención a través de consultorios externos, hospitalización y servicios apoyo (farmacia,

laboratorio, ecografías) requieren mejorar sus procedimientos a través de protocolos de atención?

\begin{tabular}{lrrr} 
& Frecuencia & Porcentaje & $\begin{array}{r}\text { Porcentaje } \\
\text { acumulado }\end{array}$ \\
\hline \hline SI & 48 & 53,3 & 53,3 \\
\hline \hline NO & 24 & 26,7 & 80,0 \\
\hline \hline TAL VEZ & 6 & 6,7 & 86,7 \\
\hline \hline $\begin{array}{l}\text { NO SABE/ NO } \\
\text { OPINA }\end{array}$ & 12 & 13,3 & 100,0 \\
\hline \hline Total & 90 & 100,0 & \\
\hline \hline
\end{tabular}

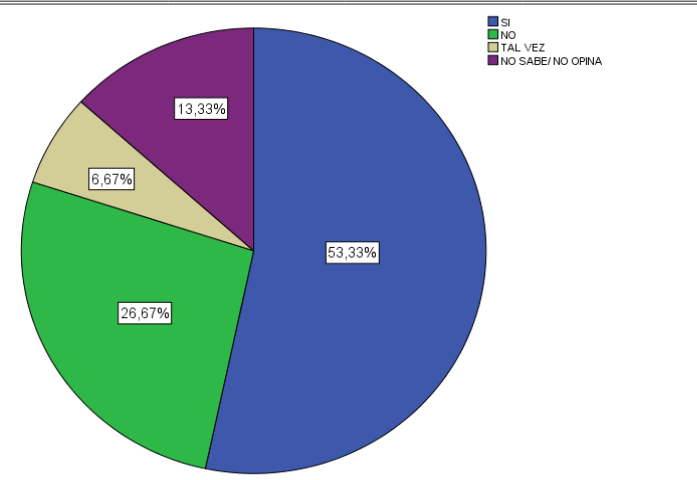

La información muestra que el $53 \%$ de los encuestados afirman que para mejorar la atención en consultorios Externos, Hospitalización y Servicios de Apoyo es nece- sario mejorar sus procedimientos a través de protocolos de atención, un $27 \%$ restante opina que no, el $13 \%$ no sabe/ no opina y el $7 \%$ tal vez.

\section{Pregunta 14}

¿Cree que la gestión del Sistema Integral de Salud permite que la atención a los beneficiarios sea oportuna?

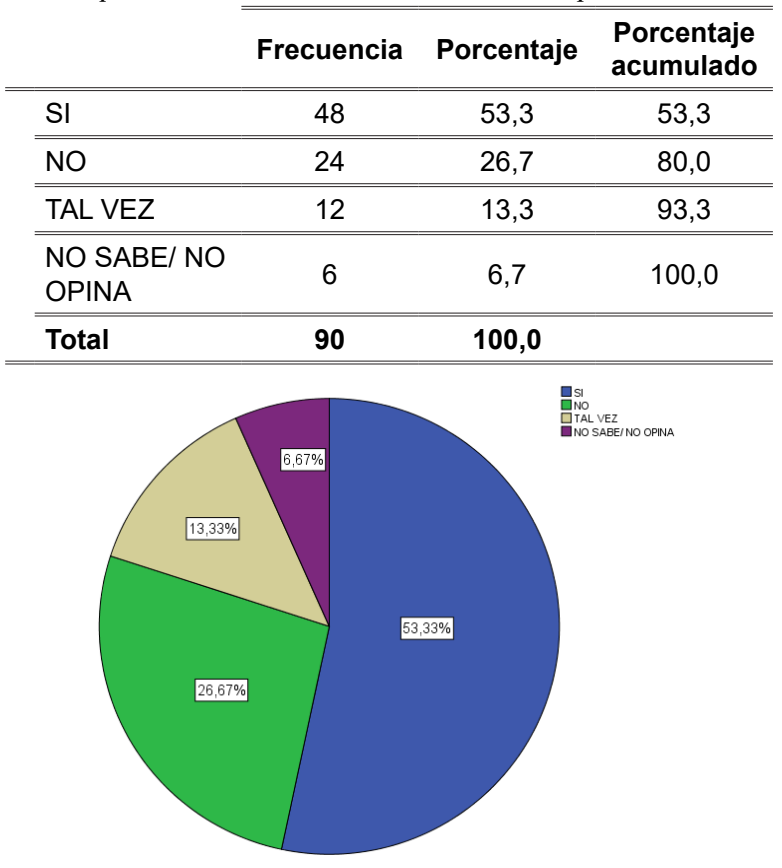

El $53 \%$ afirma que la gestión del SIS permite que la atención a los beneficiarios sea oportuna, un $7 \%$ restante no, el $7 \%$ no sabe/ no opina y el $13 \%$ tal vez.

\section{Pregunta 15}

¿Cree que las acciones o actividades de control que realizan los Órganos del Sistema Nacional de Control ayuda a mejorar la gestión del SIS?

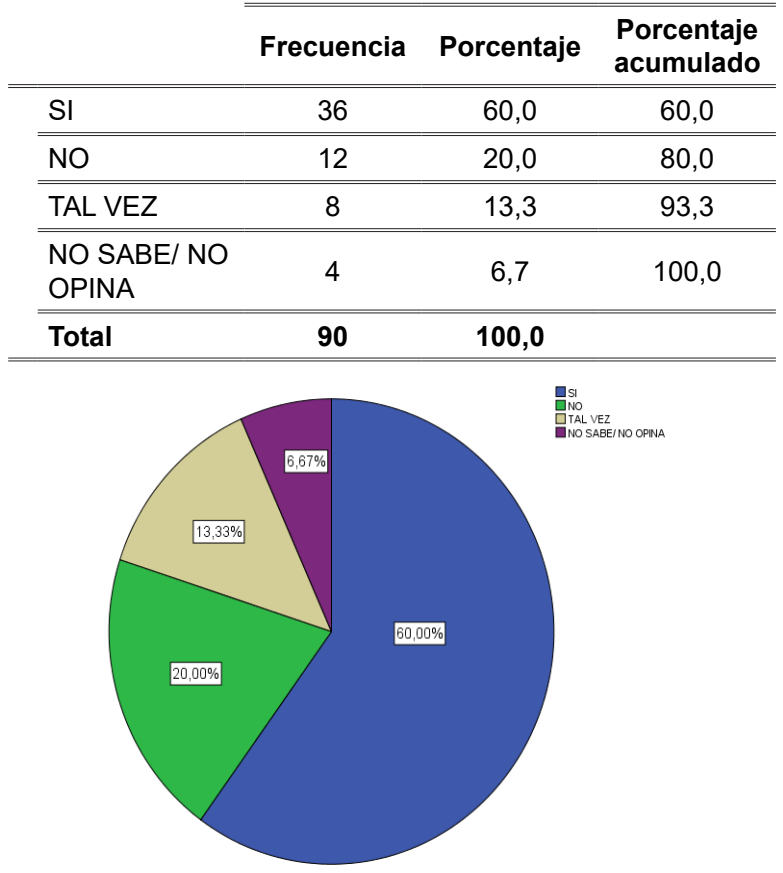


El $60 \%$ de los encuestados afirman que las acciones o actividades de control que realizan los órganos del Sistema Nacional de Control ayuda a mejorar la gestión del SIS, un $20 \%$ restante no, el $7 \%$ no sabe/ no opina y el $13 \%$ tal vez.

\section{Discusión}

El nivel de la implementación de las políticas de control interno, está en función a los niveles de cumplimiento según los elementos de control interno gubernamental en las actividades del Área de Seguros del Sistema Integral de Salud donde el nivel de implementación de las políticas vía procedimientos, protocolos, aún no son del todo satisfactorias, la percepción de los jefes y trabajadores es del 50 \% que si es importante, conocen creen que mejorará y las personas que dudan de la implementación suman alrededor del $25 \%$ y los que no se involucran otro $25 \%$, razón para que el cumplimiento total de normas si son necesarias para ayudar a mejorar la gestión del SIS. La dirección, funcionarios y trabajadores de seguros, observan que los esfuerzos emprendidos aún son muy débiles y que a ese ritmo donde no se han emitido y difundido las normas en el marco del Sistema de Control Interno, hay falencias y se evidencia en el diagnóstico que parte desde la selección de beneficiarios, existen los procedimientos en los primeros niveles de atención, sin embargo al llegar al hospital la atención hecha no es reembolsada porque pasan por otros procesos los mismos que no se encuentran debidamente normados o escapan los controles. Que el incremento de personal médico para las labores de auditoria de historias clínicas se torna relevante ya que constituye el filtro para recibir el $100 \%$ de las atenciones mediante las transferencias presupuestarias que corresponden al reembolso de todo lo gastado en cada paciente atendido, un $50 \%$ del personal de hospitalización, consultorios externos y servicios de apoyo están a favor de implementar procedimientos, protocolos para dar una mejor atención y un $60 \%$ del personal esta consiente que se tienen que realizar más actividades y acciones de control.

\section{Conclusiones}

La implementación de los procedimientos de control interno ayuda a mejorar la gestión del Sistema Integral de Salud del Hospital Regional Docente Materno Infantil El Carmen y contribuye en la elaboración de manuales de procedimientos y protocolos a nivel de áreas que requieren especial atención en la gestión de los recursos manejados a través del SIS, lo que permitirá el recupero del 100\% de las atenciones de beneficiarios a través de las transferencias presupuestarias, tal implementación debe ser retroalimentado a través de cuestionarios de control interno a fin de poder analizar las deficiencias en el manejo del SIS y aplicar los correctivos mediante el diseño de nuevos procedimientos enmarcadas en políticas hospitalarias

El diagnóstico del área de seguros, nos permitió conocer los riesgos y debilidades del SIS en el Hospital Regional Docente Materno Infantil El Carmen, impregnado en el quehacer diario de los trabajadores quienes están comprometidos con la implementación del control interno, para mejorar los procesos del SIS de manera progresiva Se vienen diseñando a través de actividades que agregan valor a las atenciones, protocolos y registros en general en el marco de las Normas de Control Interno existentes para los organismos gubernamentales, con el fin de convertirlos como parte de los procedimientos válidos en la gestión del SIS, mediante la aplicación en los casos encontrados como resultado para que optimice las tareas realizadas.

\section{Recomendaciones}

Se deberá sistematizar la aplicación de cuestionarios por áreas del control interno como mínimo cada trimestre, a fin de evaluar la gestión del Sistema Integral de Salud del Hospital Regional Docente Materno Infantil El Carmen y aplicar los correctivos pertinentes en concordancia a las normas de control interno vigentes; y que el Órgano de control institucional del hospital realice el constante monitoreo en el desarrollo de la implementación del control interno.

Se deberá implementar las acciones y procedimientos necesarios para el cumplimiento de las normas del Sistema de Control Interno para el Sector Público, establecido en la Resolución de Contraloría $\mathrm{N}^{\circ}$ 320-2006-CG y seguir con la evaluación del sistema de control interno, a fin de mejorar sus deficiencias y mejorar continuamente sus normas de control interno aplicables, a las actividades y funciones del área de Seguros, según la Resolución de Contraloría $N^{\circ}$ 320-2006-CG, y la Guía de implementación aprobado mediante Resolución de Contraloría $\mathrm{N}^{\circ}$ 458-2008-CG, ya que es un instrumento que guía el proceso de implementación del Sistema de Control Interno.

Se recomienda la emisión de directivas o normas internas en todas las oficinas, departamentos, áreas para la gestión del Sistema Integral de Salud, que regularicen detalladamente las fases y procesos que se realizan para atención de los afiliados debiendo elaborarse planes de trabajo para el cumplimiento de objetivos.

\section{Referencias bibliográficas}

Claros, R. \& León O. (2012) El control interno como herramienta de gestión y evaluación (1 era ed.) Perú: Editores Pacifico 
Cisneros, C. \& Hernández, M. \& Hernández, L. (2010) Diseño de un sistema de control interno en base al enfoque COSO ERM para el manejo de inventarios en el proceso de elaboración y venta de alimentos en entidades de Beneficencia Pública (1era ed.) San Salvador: Universidad de El Salvador

Campos, C. (2003) Los procesos de control interno en el departamento de ejecución presupuestal de una institución del Estado (1era ed.) Perú: Universidad Nacional Mayor de San Marcos.

Estrella, H. \& Munive, R.( 2004) Auditoría - Organización de papeles de trabajo en el proceso integral de un examen especial de auditoria gubernamental (1 era ed.) Huancayo: Ediciones San Francisco.

Vílchez, D. (2008) Control interno y su incidencia en la ejecución de gastos en la Municipalidad Provincial de Huamanga (1era ed.) Ayacucho: Universidad San Cristóbal de Huamanga

Contraloría General de la República (2002). Ley $N^{o}$ 27785 Ley Orgánica del Sistema Nacional de Control y de la Contraloría General de la República (1era ed.) Perú: CGR

Contraloría General de la República (2006). Ley $N^{o}$ 28716 Ley de Control Interno de las entidades del Estado (1era ed.) Perú: CGR

Contraloría General de la República (2006). Resolución de Contraloría General No 320-2006-CG Normas de Control Interno (1 era ed.) Perú: CGR

Contraloría General de la República (2006). Guía para la implementación del Sistema de Control Interno de las entidades del Estado. Resolución de Contraloría General No 458-2008-CG

\section{Referencias Electrónicas:}

Auditol (www.auditol.com).

Contraloría General de la República.(www.contraloría. gop.pe)

Organismo Superior de las Contrataciones del Estado. (www.osce.gop.pe 